\title{
ARQ MECHANISM IN HSDPA
}

Two principal features of the MAC-hs protocol include retransmissions of erroneous blocks and in-sequence data delivery to the upper layer. The first function is provided through a HARQ mechanism. The second function is achieved with the help of sliding transmission/receiving window and by using a specific numbering. In this paper, the MAC-hs performance for different sliding window sizes, timer values and number of retransmission attempts are investigated. Simulations show that values of these parameters have to be carefully set up in order to prevent incorrect block discards at the receiver side.

\section{Introduction}

The HSDPA concept (High Speed Downlink Packet Access, e.g., [1], [2]) of UMTS (Universal Mobile Telecommunication system) was introduced in Release 5 of $3 \mathrm{GPP}$. The HSDPA includes several enhanced techniques such as fast link adaptation, Hybrid ARQ (HARQ) or higher order modulation (16QAM). The enhancements make it possible to increase downlink data rates up to $10 \mathrm{Mbit} / \mathrm{s}$ on the air interface.

The HSDPA introduces a new transport channel and three physical channels (see Fig. 1). The transport channel, High Speed Downlink Shared Channel (HS-DoShCH), is shared among several users. The HSDPA scheduler reallocates radio resources, i.e. channelization codes, with a period called HS-DoShCH TTI (Transmission Time Interval). For the UMTS FDD mode, the HS-DoShCH TTI is set to $2 \mathrm{~ms}$ ([2]). Within a HS-DoShCH TTI, radio resources can be allocated to one or several users.

At the physical level, data of HS-DoShCH (i.e. MAC-hs PDUs) is mapped into a frame structure of HS-Physical Downlink Shared Channel (HS-PDoShCH). Three consecutive HS-PDoShCH slots form a radio unit for traffic. We denote this three-slot unit as T-slot. The T-slot duration corresponds to the HS-DoShCH TTI duration. One HS-PDoShCH equals one channelization code. Up to 15 channelization codes ([3]) can be employed, i.e. up to 15 HSPDoShCHs can be assigned in one T-slot. The signaling is carried by a downlink HS-Shared Control Channel (HS-ShCoCH).

HSDPA signaling information (downlink/uplink) is conveyed via control channels. The downlink signaling informs a mobile how to decode transmitted data on the HS-PDoShCHs (type of modulation and coding, transport format, HARQ information).

The downlink signaling is transported by HS-Shared Control Channel (HS-ShCoCH), whereas the uplink signaling (HARQ Ack/NAck and Channel Quality Indication) is carried by HS-Dedicated Physical Control Channel (HS-DePCoCH). The transmis-
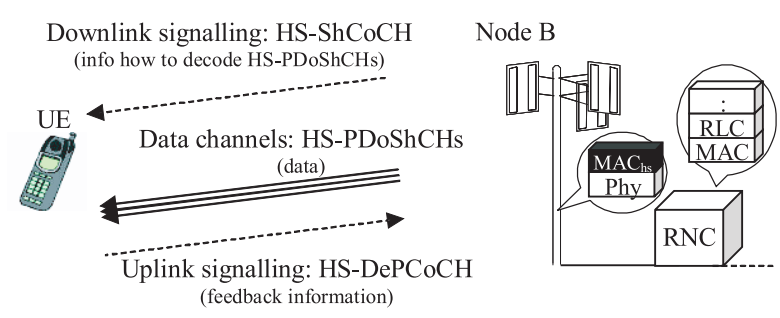

Fig. 1 HSDPA physical channels: HS-PDoShCHs (High Speed Physical Downlink Shared Ch.), HS-ShCoCH (HS Shared Control Ch.), HS-DePCoCH (HS Dedicated Physical Control Ch.).

sion of HS-ShCoCH precedes HS-PDoShCH by $1.33 \mathrm{~ms}$ (or 2 slots, [1]).

The uplink signalling (HARQ Ack/NAck and Channel Quality Indication, or CQI) is carried by HS-Dedicated Physical Control Channel (HS-DePCoCH).

The scheduling and HARQ functions are implemented in a new MAC entity (Medium Access Control) called MAC-hs (MAC-high speed, e.g., [4]). The MAC-hs is located in the Node B (Fig. 1). The MAC-hs layer (or entity) can be regarded as a layer composed of two sub-layers: the upper one and the lower one.

The lower MAC-hs sub-layer handles data (re)transmissions between the Node B and UE (User Equipment). The HARQ mechanism is based on an ARQ method: Stop and Wait. Up to 8 independent HARQ processes or instances can be simultaneously activated per UE ([4]), i.e. up to 8 MAC-hs PDUs can be handled at the same time. We denote MAC-hs PDUs as d-Blocks in the rest of paper. At most one HARQ process per UE can be activated in a T-slot.

The upper MAC-hs sub-layer manages flow control, reassembling/segmentation, numeration, and in-sequence data delivery to

\footnotetext{
* Robert Bešták

Department of Telecommunications Engineering, Faculty of Electrical, Engineering, Czech Technical University in Prague, Technicka 2, 16000 Prague 6, Czech Republic, tel. 00420-224 355 996, e-mail: bestar1@fel.cvut.cz
} 
the upper layer. The in-sequence function is fulfilled by using a specific numbering and by using sliding transmission/receiving window. This paper investigates the MAC-hs performance for different window sizes and for different values of reordering release window timer (designated in $3 \mathrm{GPP}$ specification as timer T1).

The rest of the paper is organized as follows. The next section describes the MAC-hs protocol with the focus on the in-sequence data delivery function. The simulation model is presented in section III. Section IV describes simulation scenarios and results. The last section presents our conclusions.

\section{MAC-hs protocol}

The HSDPA can activate up to 8 priority queues per MAC-hs entity (per UE). The pot of HARQ processes is shared by all active queues. Each time a new d-Block is sent, the MAC-hs scheduler determines: UE, priority queue and a suitable d-Block size. The d-Block size selection is important since the size cannot be modified during retransmission attempt(s). A proposal dealing with this issue can be found in [5].

A d-Block is assigned a Transmission Sequence Number (TSN, modulo 64), Queue Id ( 3 bits) and HARQ Id ( 3 bits). The TSN and QId are carried in the MAC-hs PDU header, whereas HARQId is carried by HS-ShCoCH. Each priority queue manages numbering of d-Blocks (i.e. TSN) independently to other priority queues.

The assigned HARQ process controls d-Block (re)transmissions. If a retransmission occurs, the original d-Block size is kept constant and different MCSs (Modulation Coding Scheme) may be used. Different MCSs lead to different coded block sizes. We denote coded blocks as c-Blocks; a c-Block corresponds to a dBlock after applying channel coding.

The sorting of received d-Blocks is provided in a MAC-hs reordering entity. The scheduler may only send d-Blocks with TSN that lie within the sliding MAC-hs transmission window.

Notice that due to the multi-instance ARQ feature together with the specific numbering of d-Blocks, the MAC-hs retransmission mechanism behaves as if the ARQ scheme Selective Repeat were used.

D-Blocks that become out-of-date are discarded from the transmission buffer. No explicit signaling is done between the Node and UE when discarding data. The receiver is informed about a discard event implicitly: either (i) by expiration of the re-ordering release timer or (ii) by receiving a d-Block above the upper edge of the receiving window. We denote the first discard as timer discard and the second one as window discard.

The re-ordering release timer is called timer $\mathrm{T} 1$. The timer controls stall avoidance events in the UE reordering buffer. There is one timer per receiving priority queue. The $\mathrm{T} 1$ is initialized for a d-Block that cannot be delivered to the upper layer due to previ- ous missing d-Block(s) in the MAC-hs reordering entity (e.g.; dBlock with TSN $=\mathrm{x}, \mathrm{d}-\mathrm{B}\left(\operatorname{lock}_{\mathrm{x}}\right)$. The T1 is stopped as soon as the $\mathrm{d}$-Blockx is delivered to the upper layer.

If the $\mathrm{T} 1$ expires, the receiving window is advanced in such a way that: a) all correctly received data up to d-Blockx (inclusive) and b) all correctly in-sequence received data above d-Block $\mathrm{x}_{\mathrm{x}}$ are delivered to the upper layer. If there is still a d-Block in the reordering entity that cannot be delivered to the upper layer, the $\mathrm{T} 1$ is restarted for that d-Block.

The receiving window is also updated and data discarded if a d-Block above the upper edge of the receiving window is obtained (i.e. the window discard occurs). The received d-Block forms a new upper edge of the window. If necessary, the $\mathrm{T} 1$ is activated in the same way as in case the of timer discard.

\section{Model of simulation}

A fixed number of UEs $(=10)$ is considered in the cell. The simulation model and layer architecture are illustrated in the figure below (Fig. 2).
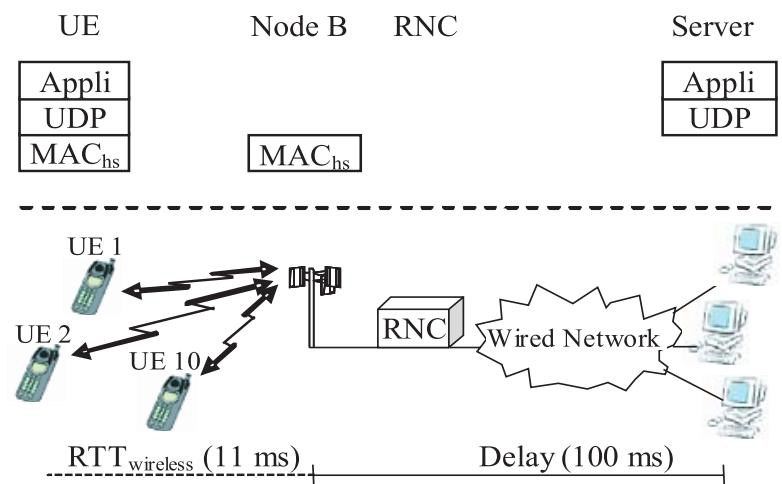

Fig. 2 Model of simulation.

MAC-hs scheduler. The scheduler is based on the Round Robin algorithm. The maximum number of HSDPA channelization codes that can be assigned in one T-slot is 12 .

Radio conditions and MCSS. The variation of radio channel conditions is simulated via a variable SIR (Signal to Interference Ratio). The SIR follows a normal distribution $\mathrm{N}\left(\mu, \delta^{2}\right)$, where the mean $\mu=0$ and the standard deviation $\delta=4 \mathrm{~dB}$. The memory of the random process indicates a parameter $T_{v}, T_{v} \in(20 \mathrm{~ms} ; 50$ $\mathrm{ms} ; 100 \mathrm{~ms}, 400 \mathrm{~ms} ; 1.5 \mathrm{~s}$ ). Table 1 shows the used MCSs and dBlocks sizes.

ARQ. A MCS is selected in such way that $\operatorname{SIR}($ MCS) $<$ $<$ SIR $_{\text {NodeB }}$, where SIR $_{\text {NodeB }}$ ' is the last known value of SIR in Node B for the given UE. The SIR(MCS) threshold values are given in Table 2 ([6]). 


\begin{tabular}{|c|c|c|c|c|c|c|}
\hline \multirow[b]{2}{*}{$\begin{array}{c}\text { Chann. } \\
\text { codes }\end{array}$} & \multicolumn{6}{|c|}{ Size of MAC-hs PDU (data rates), MCSs } \\
\hline & $\begin{array}{c}480 \text { bits } \\
(240 \mathrm{~kb} / \mathrm{s})\end{array}$ & $\begin{array}{c}720 \text { bits } \\
(360 \mathrm{~kb} / \mathrm{s})\end{array}$ & $\begin{array}{c}960 \mathrm{bits} \\
(480 \mathrm{~kb} / \mathrm{s})\end{array}$ & $\begin{array}{c}1440 \text { bits } \\
(720 \mathrm{~kb} / \mathrm{s})\end{array}$ & $\begin{array}{c}1920 \text { bits } \\
(960 \mathrm{~kb} / \mathrm{s})\end{array}$ & $\begin{array}{c}2880 \text { bits } \\
(1,44 \mathrm{Mb} / \mathrm{s})\end{array}$ \\
\hline 2 & $\begin{array}{c}\text { QPSK 1/4 } \\
\text { (MCS4) }\end{array}$ & $\begin{array}{c}\text { QPSK 1/3 } \\
\text { (MCS5) }\end{array}$ & $\begin{array}{c}\text { QPSK 1/2 } \\
\text { (MCS6) }\end{array}$ & $\begin{array}{c}\text { QPSK 3/4 } \\
\text { (MCS7) }\end{array}$ & $\begin{array}{c}\text { 16QAM1/2 } \\
\text { (MCS8) }\end{array}$ & $\begin{array}{c}\text { 16QAM 3/4 } \\
\text { (MCS9) }\end{array}$ \\
\hline 4 & $\begin{array}{c}\text { QPSK 1/8 } \\
\text { (MCS2) }\end{array}$ & $\begin{array}{c}\text { QPSK 0,18 } \\
\text { (MCS3) }\end{array}$ & $\begin{array}{c}\text { QPSK 1/4 } \\
\text { (MCS4) }\end{array}$ & $\begin{array}{c}\text { QPSK 1/3 } \\
\text { (MCS5) }\end{array}$ & $\begin{array}{c}\text { QPSK 1/2 } \\
\text { (MCS6) }\end{array}$ & $\begin{array}{c}\text { QPSK 3/4 } \\
\text { (MCS7) }\end{array}$ \\
\hline 6 & $\begin{array}{c}\text { QPSK 0,08 } \\
\text { (MCS1) }\end{array}$ & $\begin{array}{c}\text { QPSK 1/8 } \\
\text { (MCS2) }\end{array}$ & $\begin{array}{c}\text { QPSK 1/8 } \\
\text { (MCS2) }\end{array}$ & $\begin{array}{l}\text { QPSK 1/4 } \\
\text { (MCS4) }\end{array}$ & $\begin{array}{l}\text { QPSK 1/3 } \\
\text { (MCS5) }\end{array}$ & $\begin{array}{c}\text { QPSK 1/2 } \\
\text { (MCS6) }\end{array}$ \\
\hline
\end{tabular}

SIR thresholds for different MCSs.

\begin{tabular}{|c|c|c|c|c|c|c|c|c|c|}
\hline & MCS1 & MCS2 & MCS3 & MCS4 & MCS5 & MCS6 & MCS7 & MCS8 & MCS9 \\
\hline SIR [dB] & -12 & -7 & -5 & -4 & -1 & 1 & 3 & 5 & 9 \\
\hline
\end{tabular}

Due to feedback delay and scheduling, there is delay between the fresh SIRNodeB value and the moment of selecting MCS. The minimum (maximum) delay is set to be $6 \mathrm{~ms}(20 \mathrm{~ms})$. When transmitting a new d-Block, the selected MCS can correspond to several d-Block sizes and the lowest one is chosen. The chosen MCS and d-Block size determine the number of channelisation codes that need to be used. Within retransmissions, MCSs are chosen from the column of corresponding d-Block size.

A HARQ instance in UE processes a c-Block according to the following procedure:

if $\mathrm{SIR}(\mathrm{MCS})<\mathrm{SIR}_{\mathrm{UE}}$ then erroneous c-Block

else correctly decoded c-Block,

where $\mathrm{SIR}_{\mathrm{UE}}$ gives the current value of SIR measured in the UE. The maximum number of retransmissions per c-Block is delimited by a parameter denoted in our paper as MaxDat; MaxDat $=2$. The downlink and uplink signaling is assumed to be error free.

Traffic model. Every UE uses web-browsing running over UDP (User Data Protocol). A web-browsing session comprises of several packet calls or web pages. A packet call is followed by a reading time interval to view the download contents. The packet call size is modeled by Pareto random variable, with cutoff ( $\alpha=1.6$; $\min =1.8 \mathrm{kB} ; \max =40 \mathrm{kB}$, mean $=4.4 \mathrm{kB}[7])$. The reading time follows an exponential random variable $($ mean $=5 \mathrm{~s})$.

\section{Simulation}

Simulation experiments are carried out for two MAC-hs sliding window sizes (4 and 16). In the Node B, a d-Block is discarded whenever a retransmission counter associated with c-Block reaches the value of MaxDat. In a UE, a d-Block is discarded either due to the timer discard or due to the window discards. Fig. 3 and Fig. 4 show a ratio of discarded d-Blocks in the Node B versus discarded d-Blocks in UEs (1).

For small values of $\mathrm{T} 1$ (20, 50, $100 \mathrm{~ms})$, a UE discards more d-Blocks than the Node B does. A missing d-Block in a UE is discarded before the erroneous c-Block can be corrected through the retransmission mechanism. For higher values of T1 (400, $500 \mathrm{~ms}$ ), the number of discarded d-Blocks in the Node B and UEs is the same. As the variation of channel conditions slows down (values of $T_{v}$ increase), the ratio (1) gets smaller for the $T 1=20 \mathrm{~ms}$. For higher values of $T 1$ (50, $100 \mathrm{~ms})$, the ratio (1) increases.

Larger MAC-hs window size has little impact on the number of discarded d-Blocks (Fig. 4); graphs in Fig. 3 and Fig. 4 are about the same.

Let's now investigate which of the UE's discard mechanisms dominates: timer discard or window discard. The ratio of discarded d-Blocks due to the window discard versus all discarded d-Blocks in UEs is shown in Fig. 5 and Fig. 6 (2).

$$
\begin{gathered}
\sum_{\text {NodeB }} \text { discarded blocks duet to the Max Dat discard } \\
\sum_{U E} \text { discarded blocks due to the timer discard }+\sum_{U E} \text { discarded blocks due to the window discard } \\
\sum_{U E} \text { discarded blocks due to the timer discard }+\sum_{U E} \text { discarded blocks due to the window discard }
\end{gathered}
$$


Fig. 5 shows that for $T 1=20 \mathrm{~ms}$, the timer discard dominates, no matter how fast the channel conditions change. Just a few dBlocks are discarded due to the window discard; the value of $T 1$ is so small that missing d-Blocks in UEs are discarded before the MAC-hs retransmission mechanism can take steps. For other values of $T 1$, the window discard becomes more and more important as the variation of channel conditions slow down (values of $T_{v}$ increase). The discarded d-Blocks in the Node B are detected in UEs by receiving d-Blocks above the upper edge of the receiving window. When setting the window to larger size, more d-Blocks are processed at the same time. The transmission delay of d-Blocks increases and the timer discard becomes more important (Fig.6).

\section{Conclusions}

The paper studies performance of the MAC-hs protocol for different window sizes and for different values of the release window timer $\mathrm{T} 1$.
Simulations show that values of $\mathrm{T} 1$ and MaxDat have to be adequately set up. Setting up values of $\mathrm{T} 1$ too small, compared to values of MaxDat, results in more discarded d-Blocks in UEs than the Node B discards. D-Blocks are discarded in the UE due to the timer discard. In other words, unnecessary discarded d-Blocks at the MAC-hs level deteriorates received data from the user's point of view. For example, the discarded data can degrade a received audio/video signal. If a reliable data transfer is required, the unnecessary discarded data at the MAC-hs level has to be retransmitted through retransmission mechanisms of a higher layer (e.g., RLC or TCP). However, this results in an increase in transmission delay as well as in higher user cost.

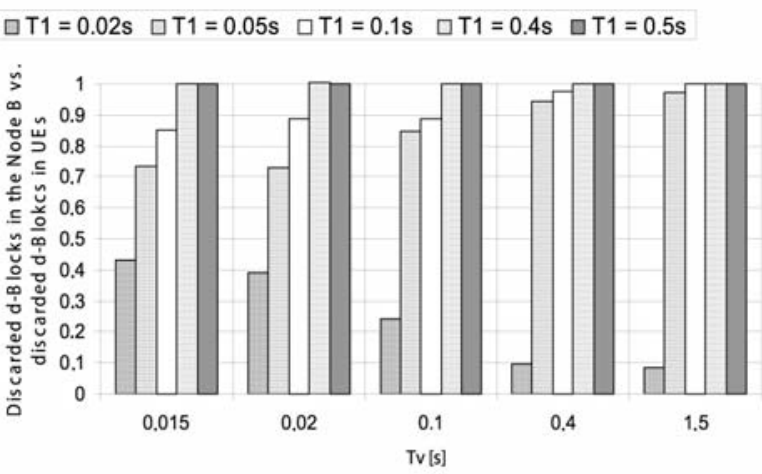

Fig. 3

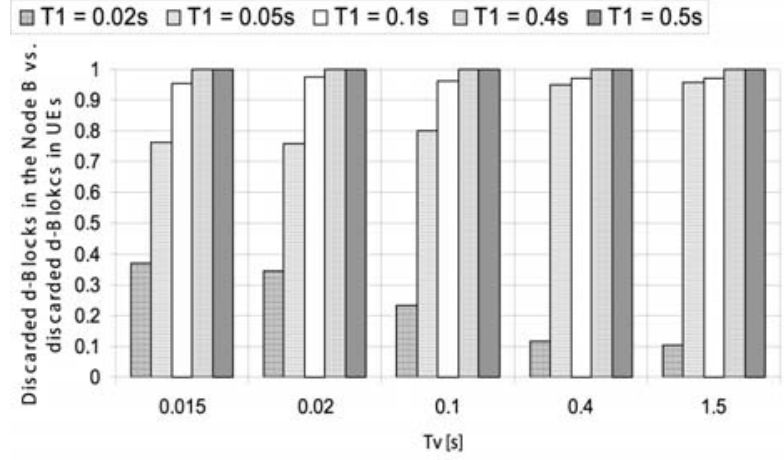

Fig. 4

Fig. 3, Fig. 4 Ratio of discarded d-Blocks in the Node B versus discarded d-Blocks in UEs, window size $=4$ (Fig. 3) or window size $=16$ (Fig.4).

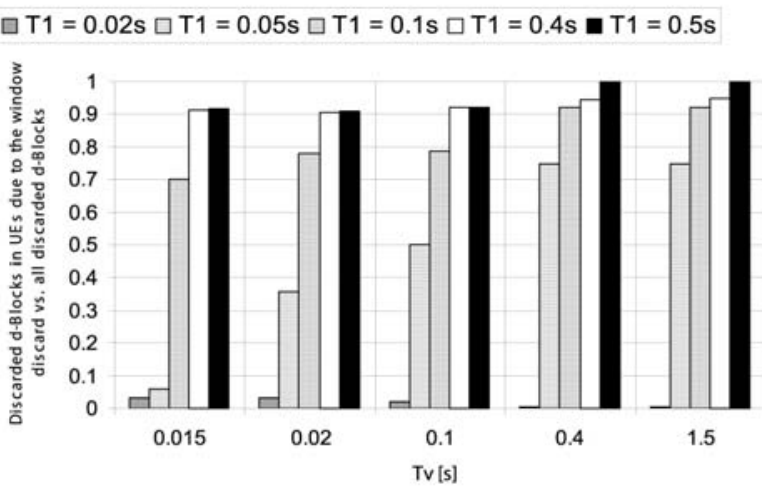

Fig. 5

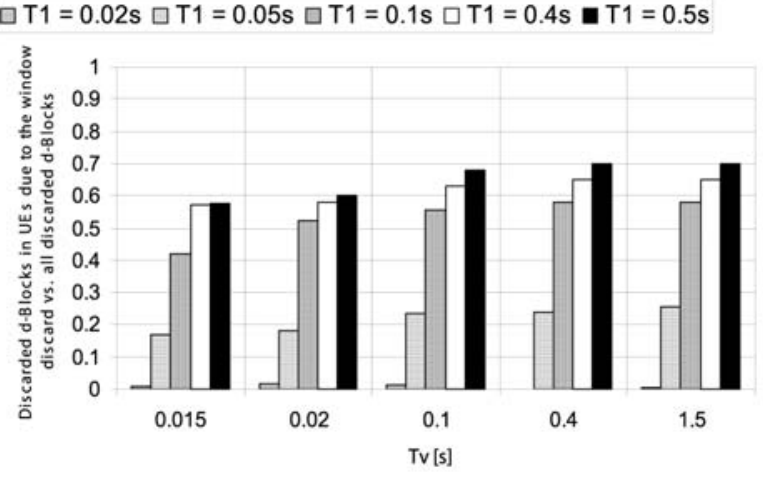

Fig. 6

Fig. 5, Fig. 6 Ratio of discarded d-Blocks in a UE due to the window discard versus all discarded d-Blocks; window size $=4$ (Fig. 5) or window size $=16$ (Fig.6). 


\section{References}

[1] HEDBERG, T., S. PARKVALL, S.: Evolving WCDMA, Ericsson review, 2/2000

[2] TS 25.308: High Speed Downlink Packet Access (HSPDA), Overall Description, Stage 2 (Release 6), 3GPP, September 2004

[3] TS 25.306: UE Radio Access capabilities (Release 5), 3GPP, September 2003

[4] TS 25.321: MAC protocol specification (Release 6), 3GPP, September 2004

[5] BESTAK, R., GODLEWSKI, P., MARTINS, P.: HSDPA adaptation scheme of MAC-hs PDU size for retransmissions, CSN 2003, Benalmadena, Spain, September 2003

[6] PARKVAll, S., PEISA, J., FURUSKÄR, A., SAMUELSSON, M., PERSSON, M.: Evolving WCDMA for Improved High Speed Mobile Internet, Proc. of the Future Telecommunications Conference 2001, Bejing, China, November 2001

[7] STACEY, M., NELSON, J., GRIFFIN, I.: TCP for Transactions, Linux journal, 47/1999. 